\title{
LA UNIVERSIDAD Y LA MOVILIZACIÓN DE CONOCIMIENTOS EN LA SOCIEDAD: EL CASO VENEZOLANO*
}

The university and knowledge mobilization in our societies: the Venezuelan case

\section{Luis A. Ordóñez Vela**}

Resumen: El paradigma del conocimiento universitario se encuentra en transformación. Partiendo de las ideas de Pierre Bourdieu de campo y habitus, y llegando a los postulados de Lev Vigotsky del compañero más capaz y la propuesta de conocimiento conectivista de Stephen Downes y George Siemens, el artículo explora los cambios que ha experimentado la universidad en su función respecto al conocimiento y la necesidad de que la misma, especialmente en Latinoamérica, se vuelva una universidad que "moviliza el conocimiento". A partir del caso de Venezuela, el autor explora los múltiples esfuerzos de insertar la ciencia y tecnología en la sociedad latinoamericana, y en sus universidades en particular, y cómo la universidad falló en convertirse en un factor de peso en la toma de decisiones de políticas públicas en nuestros países. La propuesta es que la universidad, sobre todo la universidad pública latinoamericana, debe desarrollar procesos para convertirse en una auténtica movilizadora de conocimiento con tal de responder a las demandas que las sociedades le están exigiendo.

Palabras clave: Universidad, movilización, conocimiento, sociedad, tecnología, paradigma universitario.

Este es la primera parte de dos entregas, la segunda se publicará en el cuarto número de Ciencia y Sociedad, 2015.

** Académico de la Universidad Simón Bolívar, Venezuela. Director de los Cursos Abiertos Masivos en Línea (CAMEL).Correo electrónico: lordonezv@gmail.com. 
Abstract: The paradigm of University knowledge is in transformation. Based on the ideas of Pierre Bourdieu's habitus and field, and incorporating the postulates of Lev Vygotsky's most capable partner and the proposal of connective knowledge advanced by Stephen Downes and George Siemens, the article explores the changes experienced by the University in its role regarding knowledge mobilization and the need, especially in Latin America, to evolve into a University that "mobilizes knowledge". Using the case of Venezuela, the author explores the multiple efforts to insert science and technology in our societies, particularly in its universities, and how the University has failed in becoming an important factor in the decision-making processes for public policies in the country. The proposal is that the University, especially the Latin American public University, should develop processes to become a genuine knowledge mobilizer so to respond to the demands that our societies are generating.

Keywords: University, mobilization, knowledge society, technology, university paradigm.

\section{Introducción}

El paradigma universitario ha cambiado con el tiempo. Desde la universidad organizada corporativamente entre profesores y estudiantes del siglo XII, primero en París ${ }^{1}$, para transmitir conocimiento, hasta llegar a la universidad creadora de conocimientos, concebida por Guillermo Humboldt en la Alemania del siglo XIX (Universidad

1 Aunque el primer documento que reconoció oficialmente la existencia de un grupo de maestros y estudiantes data de Bolonia en 1158. En él, Federico Barbarroja concedió jurisdicción especial, escolar y eclesiástica, a los extranjeros residentes en la Ciudad. (Muscará, 2012) 
de Berlín, 1809), tuvieron que transcurrir varios siglos. Posteriormente, las disputas del siglo XIX en el sistema universitario norteamericano entre la universidad práctica (universidad para profesionales) o teórica (universidad para intelectuales) abrieron el camino al paradigma de la universidad de extensión, que ve la luz quizás por primera vez en la universidad para la educación cooperativa del siglo XX (Universidad de Cincinnati, 1906) que, al incorporarse a los paradigmas anteriores, aún marca las estructuras organizativas de nuestras universidades (decanatos de docencia, investigación y extensión).

De nuevo, el paradigma está en transformación. Las nuevas formas de generar y movilizar conocimientos bajo el impacto de las tecnologías de información y comunicación (TIC) están produciendo una serie de situaciones sin precedente que preocupan a los interesados en la gestión universitaria, generando literatura como el libro de Taylor (2010) donde se analiza la crisis del "campus" y se proponen planes para reformar los "colleges" y las universidades del norte.

Desde esas latitudes se proponen acciones como terminar con el escalafón, reestructurar los departamentos para estimular mayor colaboración entre disciplinas, hacer énfasis en la docencia en lugar de la investigación, y enseñar en nuevas áreas del conocimiento.

Ese nuevo modo de movilizar el conocimiento que va apareciendo en la sociedad, y que lleva incluso a tener que repensar todo lo que significa movilizar información y "democratizar conocimiento" al interior de una sociedad con las repercusiones subsiguientes, se nos despliega en toda su magnitud en el "papel de trabajo" publicado en el 2012 que lleva por título Expanding our understanding of $K+$ (KT, KE, KTE, KMb, KB, KM, etc) (Shaxson et al., 2012) y que traduciría libremente al castellano como: "Expandiendo nuestra comprensión del concepto de Conocimiento plus (transferencia de conocimiento $(\mathrm{KT})$, Intercambio de conocimiento $(\mathrm{KE})$, Traducción e Intercambio de conocimiento (KTE), Movilización de conocimiento (KMb), Intermediación de conocimiento (KB), Gerencia 
de conocimiento (KM), etc.". En su definición, el Conocimiento plus de acuerdo a estos autores sería:

El término genérico que se da al conjunto de funciones y procesos en las varias interfaces entre conocimiento, práctica cotidiana y politicas públicas. El Conocimiento plus mejora la manera en las que el conocimiento es compartido y aplicado; mejorando asi los procesos existentes para generar cambios más efectivos y sustentables.

Como es natural, esta complejidad emergente obliga a revisar cómo se moviliza conocimiento entre la universidad y la sociedad.

Pero previamente, debemos examinar lo que se conoce sobre un aspecto relativamente más susceptible de análisis, aunque también sumamente complejo, que implica una pregunta: cómo se gerencia conocimiento al interior de las organizaciones, en nuestro caso de interés, al interior de la "organización universitaria".

\section{La movilización de conocimientos entre la universidad y la sociedad}

Cada día más las organizaciones luchan por hacer mejor uso del conocimiento que existe dentro de ellas.

"Es muy importante desde el comienzo marcar la diferencia entre conocimiento e información. El conocimiento está estrechamente vinculado a todas las experiencias anteriores y existe solo en el contexto de la mente. No puede ser gerenciado. Lo que puede ser gerenciado es el flujo de ese conocimiento hacia otros. Lo que puede ser transmitido es información (datos en contexto), no conocimiento... De acuerdo a la unidad de inteligencia de The Economist, la gestión del conocimiento es una de las cinco tendencias claves que determinarán competitividad en el futuro inmediato. Las otras cuatro son: globalización, demografía, atomización y personalización" (Leistner, 2010). 
En su libro Dominando el flujo de conocimiento en la organización: Cómo hacer que funcione el compartir conocimientos, Leistner (2010) propone que el éxito de cualquier actividad que pretenda gerenciar el conocimiento existente en los individuos que conforman una organización depende de cuatro elementos: el capital humano, la tecnología, los procesos y la cultura. De igual modo, en la presentación del libro, el prologuista Larry Prusak propone algunos supuestos fundamentales para la gerencia del conocimiento en organizaciones, los cuales serían:

a) El conocimiento puede ser mejor comprendido como un fenómeno social, y los esfuerzos para procesarlo están mejor estructurados cuando priorizan esfuerzos grupales que cuando son individuales.

b) El trabajo con conocimiento necesita un acercamiento que incluya una mezcla o combinación de tecnología, estrategia, capital humano y capital social.

c) Es casi imposible medir conocimiento eficientemente, y no vale la pena hacerlo.

d) Por todo lo anterior son recomendables los acercamientos holísticos al problema, no importa lo difícil que sea formularlos e implementarlos.

Es de notar cómo ambos autores mencionan los mismos cuatro elementos que se deben considerar a la hora de intentar movilizar conocimiento en una organización, a saber: Gente (capital humano de Prusak), tecnología, proceso (la estrategia de Prusak) y cultura (capital social).

Si aplicamos estos conceptos al análisis de la organización universitaria podemos asumir un alto capital humano y un importante control de las tecnologías que pudiesen ser requeridas, por ser estos elementos consustanciales al quehacer universitario. De lo que no podemos estar tan seguros es que tengamos clara conciencia 
de la cultura de la organización universitaria y cómo esta pudiese resultar en la generación de cuellos de botella que impidiese el flujo de conocimiento (el proceso) al interior de su estructura. Este problema de la cultura, unido al del paradigma universitario reinante que coloca el énfasis en la "generación" de conocimiento, pudiese incidir en la deseada pero no tan eficiente "movilización de conocimientos entre la sociedad y la universidad".

Los estudiosos de la administración le dan un sentido muy preciso a la cultura de la empresa (en tanto que una organización) a partir de los estudios pioneros de Hofstede (1997) y sus medidas de la "dimensión cultural" basadas en:

1. El índice de distancia al poder: "el grado en el cual los miembros menos poderosos aceptan y esperan que el poder sea distribuido desigualmente".

2. Individualismo: Qué tanto los individuos se integran en grupos.

3. Masculinidad: La distribución de roles emocionales entre los géneros.

4. Índice de evasión a la incertidumbre: El nivel en el que los miembros de una sociedad tratan de enfrentar la ansiedad minimizando la incertidumbre.

5. Orientación de largo plazo: "El horizonte temporal de las sociedades".

Las reglas para movilizar conocimiento en el interior de la universidad deben responder a los patrones generales que las investigaciones han ido descubriendo, entre ellos el de la cultura. Y si aceptamos que existe "una cultura latinoamericana", como ha sido demostrado abundantemente, entre otros por Inglehart y Welzel en su World $V$ alues Survey (Inglehart y Welzel, 2010), es necesario que revisemos la "cultura universitaria latinoamericana" a la hora de intentar analizar cómo se movilizará conocimiento en nuestra universidad. 
Factores culturales que tienen sus consecuencias positivas y negativas, como observamos en Roche (1987) cuando nos habla de:

Los Estados Desunidos de América-Dos sentidos tiene el título de este Editorial: uno la desunión entre el Norte (EE.UU) y el sur de nuestro hemisferio y, dos, la desunión entre todos y cada uno de los Estados del sur... En el Sur, a pesar del lenguaje, de una cultura y de una religión común, la desunión es total. Atrincherados tras la sacrosanta 'soberanía nacional', maniatados por intereses creados de naturaleza económica y separados por sistemas politicos en extremo divergentes, los Estados mantienen sus puertas estancadas, se resisten a la colaboración Sur-Sur, obtienen del Norte el 99,9 por ciento de su información, pierden en pleitos de fronteras un tiempo y un dinero, [...] más productivos fueran si se dedicaran a esfuerzos de integración; en una palabra, presentan al Norte un flanco blando y vulnerable.

Otra aproximación al análisis de la cultura organizacional universitaria nos la brindan los estudios de Pierre Bourdieu (Martínez García, 1998). Ya desde sus primeras publicaciones, (ver, por ejemplo, su trabajo junto a Passeron Los herederos: los estudiantes y la cultura, 1964), se muestra fascinado por el tema educativo, pero es de su análisis general y de sus conceptos básicos de campo y habitus que podemos hacernos una idea de lo complejo de la cultura universitaria:

El campo es una red de relaciones objetivas entre posiciones objetivamente definidas -en su existencia y en las determinaciones que ellas imponen a sus ocupantes- por su situación (situs) actualy potencial en la estructura de las distribuciones de las especies de capital (o de poder) cuya posición impone la obtención de beneficios especifficos puestos en juego en el campo y, a la vez, por su relación objetiva con las otras posiciones.

Igualmente:

El habitus es la subjetividad socializada, es la generación de prácticas que están limitadas por las condiciones sociales que las soportan, es la forma en que las estructuras sociales se graban en nuestro cuerpo y nuestra mente, y forman las estructuras de nuestra subjetividad. 
Cuando analizamos a la universidad es fácil detectar que conforma un campo claramente definido con un habitus también determinante, que va a afectar la relación de la universidad y sus miembros con el resto de la sociedad.

Si a lo anterior añadimos otro concepto de Bourdieu, como lo es el de "violencia simbólica" donde el universitario trataría de diferenciarse por su "capital cultural" en una sociedad donde un título es importante porque define un nivel elevado en la escala social, es fácil imaginar como al interior de la institución-organización universitaria es difícil compartir conocimiento pues sería ceder capital cultural, pero más difícil aún es organizarse para entregarlo a estructuras externas a la propia universidad.

Si aceptamos que procesos similares ocurren simultáneamente en otros "campos" sociales, con sus propios habitus, en una sociedad determinada, como pudiese ser el "campo político", podemos apreciar la enorme dificultad de circular conocimiento eficientemente, no solo al interior de la universidad, sino también desde esta a la sociedad. Recordemos aquí cómo la difusión del conocimiento en el mundo académico se logra fundamentalmente mediante el muy elaborado ritual de la publicación en revistas "indexadas" con árbitros a ciegas, lo cual ayuda a mantener las altas exigencias académicas, pero dificulta la socialización de esa información a otros académicos, y en gran medida al resto de la sociedad.

De lo anterior podemos concluir que la separación social en campos con habitus propios de cada sector o actividad puede convertirse en la práctica en algo diferenciador para la universidad, como ha sucedido con la "cartelización" de los partidos políticos de acuerdo a Katz y Mair (1995); así, en los partidos políticos, luego de una etapa inicial donde los miembros del mismo son parte de la sociedad y del estado, con el tiempo se convierten en parte del estado y pierden contacto efectivo con la sociedad que pretenden representar. En una visión negativa de la universidad, los universitarios podrían sufrir un proceso acelerado de diferenciación de 
su campo académico con relación al resto de la sociedad, y del estado, que lleve en la práctica a la institución a separarse de estas (la sociedad y el estado), quedando solo débilmente vinculada al estado por su conocida dependencia presupuestaria (ver figura $\mathrm{N}^{\circ}{ }^{\circ}$ ).

\section{Figura N. ${ }^{\circ} 1$}

Consecuencia negativa de la separación campos en una sociedad (elaboración propia a partir de Katz y Mair, 1995)
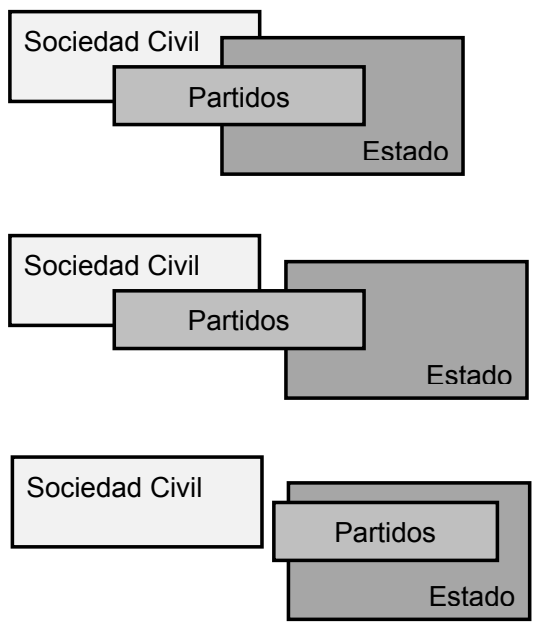

La cartelización del sistema político

Después de Katz y Mair, en Changing models of party organization and party democracy

Party Politics, 1,1,5(1995)

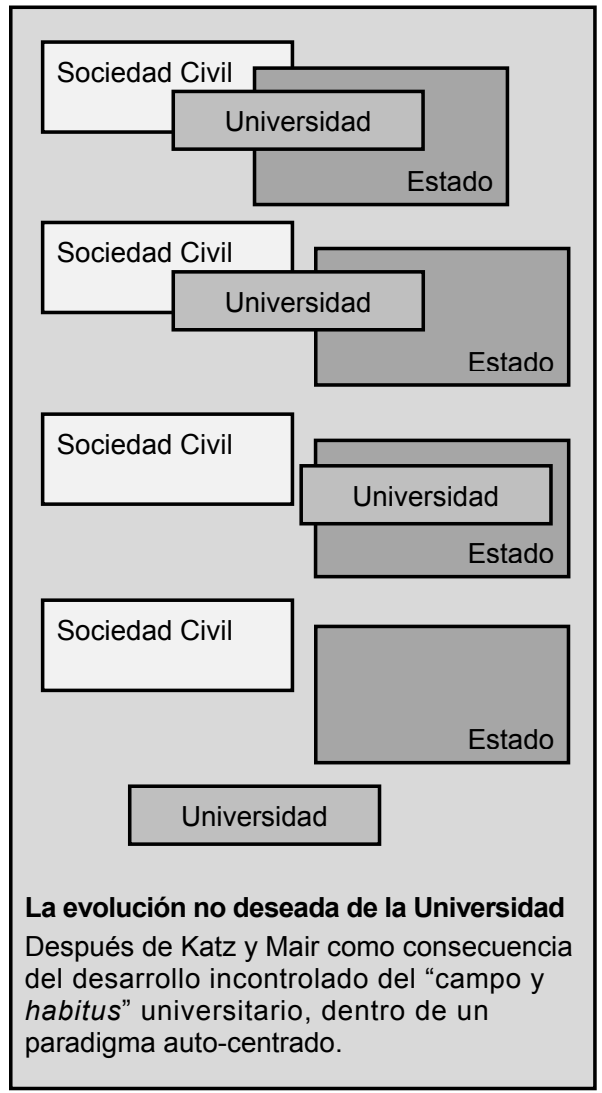

En la figura se ilustra un modelo "negativo" del proceso que puede seguir la "organización universitaria" si solo operan los factores culturales descritos por Bourdieu y no hubiere necesidad de interacción con la sociedad. "La distinción cultural no es más 
que una forma encubierta de dominación a la que denominó 'complicidad ontológica' entre el campo y el habitus", nos dice el prologuista de su obra en español La distinción: Criterio y bases sociales del gusto (1979). De allí que pudiéramos especular que una universidad que solo requiere presupuesto de parte del Estado puede generar una "cultura" autoexcluyente que impediría la movilización adecuada del conocimiento entre ella y esa sociedad. La primera consecuencia es antidemocrática, dado que la sociedad no es solo el Estado, una universidad autosegregada niega información y conocimientos al ciudadano, quien ve así dificultarse sus posibilidades de estrechar su distancia al poder, e incrementa sus ansiedades frente a la incertidumbre, con la subsiguiente aceptación de metas a corto plazo ya que no puede controlar su entorno en forma racional.

A fin de resolver esta separación entre "estructuras" que dejan de comunicarse, las ciencias administrativas han generado una serie de mecanismos, entre los que vale la pena destacar las estructuras para la "intermediación". En el campo específico de la Ciencia y Tecnología (Cy'T) destacan los estudios de Meulen (1998), que ha analizado en Europa situaciones como la del sistema nacional de investigación de los Países Bajos (Netherlands) donde ejemplifica las características generales de los sistemas de investigación modernos con presión creciente por relevancia de la ciencia, y que cuentan con un nivel intermediario entre el estado y las organizaciones que ejecutan investigación. ${ }^{2}$ Este autor destaca la competencia institucional en los procesos de agregación (heterogénea) para la construcción de agendas, así como la implantación de consorcios de acción (consociational), al tiempo que describe consejos asesores sectoriales, los ejercicios de prospectiva y los programas de

2 "Para analizar la dinámica detrás de estos procesos es útil distinguir entre los altos niveles de gobierno, un nivel intermedio de consejos de investigación, organismos financiadores, cuerpos asesores o programadores, organizaciones de universidades y otros organismo de investigación, separados a su vez del nivel que realiza las investigaciones propiamente dicho.” (Meulen, 1998). 
investigación estratégica. Lo dicho anteriormente forma parte de una gama de herramientas que buscan cerrar la brecha entre los sistemas que realizan investigación y los niveles de decisión de políticas públicas.

Siempre dentro de la misma visión, los estudios desarrollados por el mismo Meulen (ver su trabajo del 2003) en la serie de relaciones que se establecen entre los gobiernos y las agencias o consejos de fomento de la investigación y entre estas y los científicos, pero esto se entiende desde la perspectiva de la teoría de agente-principal ${ }^{3}$. En el caso de Noruega, el sistema de Cy'T estudiado por el autor, concluye que el nuevo papel para los Consejos de Investigación es el de intermediar la relación agente-principal.

De manera que para entender cómo se genera y se moviliza conocimiento en la universidad y hacia la sociedad es necesario conocer no solo cómo ocurrió el proceso de institucionalización de CyT en la universidad, sino también cómo dicho proceso pudo profundizar el fenómeno sociológico descrito por Bourdieu, y que afecta instancias tan disímiles como las instituciones artísticas o los partidos políticos, y aún más allá, debemos estudiar las estrategias gerenciales que se han venido desarrollando para generar una "intermediación" más eficiente entre los niveles decisores de políticas y los investigadores universitarios en nuestro caso. $\mathrm{Si}$ a lo anterior unimos todo el problema que se deriva de la gestión del conocimiento al interior de la propia organización, podemos comenzar a percibir la verdadera dimensión del problema y lo cada vez más complejo de los análisis que pretenden comprenderlo (K+ por ejemplo, Shaxson et al., 2012).

3 En economía, el problema del agente-principal designa un conjunto de situaciones que se originan cuando un actor económico (el principal o el jerarca), depende de la acción o de la naturaleza o de la moral de otro actor (el agente), sobre el cual no tiene perfecta información. En otras palabras, ese asunto concierne las dificultades que se presentan bajo condiciones de información asimétrica, cuando el principal contrata a un agente. (recuperado de http:/ / es.wikipedia.org/wiki/Problema_del_agente-principal el 14 de abril de 2014). 
Es precisamente dentro de esta óptica y con esta urgencia de no llegar a la situación negativa recién mencionada que nos encontramos en la búsqueda de cómo dar acceso a todos al conocimiento universitario. Para ello, introducimos en nuestro análisis los conceptos del psicólogo Lev Vygotsky y los planteamientos del "conocimiento conectivista" de la escuela canadiense de educación liderada por Siemens y Downes.

\section{El conocimiento conectivista}

De acuerdo con la visión de Vygotsky:

"El ser humano es ante todo un ser culturaly esto es lo que establece la diferencia entre el ser humano y otro tipo de seres vivientes, incluyendo los primates. Elpunto central de esta distinción entre funciones mentales inferiores y superiores es que el individuo no se relaciona unicamente en forma directa con su ambiente, sino también a través de y mediante la interacción con los demás individuos. La psicología propiamente humana es un producto mediado por la cultura. Podría decirse que somos porque los demás son. En cierto sentido, somos lo que los demás son".

Esta visión se traduce como la Zona de desarrollo próximo: "la distancia entre el nivel de desarrollo efectivo del alumno (aquello que es capaz de hacer por si solo) y el nivel de desarrollo potencial (aquello que sería capaz de hacer con la ayuda de un adulto o un compañero más capaz), sirve para delimitar el margen de incidencia de la acción educativa". ${ }^{4}$ Si nos imaginamos a la

4 "Desde esta perspectiva, la zona de desarrollo próximo es la posibilidad de los individuos de aprender en el ambiente social, en la interacción con los demás. Nuestro conocimiento y la experiencia de los demás es lo que posibilita el aprendizaje; consiguientemente, mientras más rica y frecuente sea la interacción con los demás, nuestro conocimiento será más rico y amplio. La zona de desarrollo próximo, consecuentemente, está determinada socialmente. Aprendemos con la ayuda de los demás, aprendemos en el ámbito de la interacción social y esta interacción social como posibilidad de aprendizaje es la zona de desarrollo próximo” Romo (s/f). 
universidad como el "compañero más capaz", podemos visualizar un camino para establecer y reestablecer esos vínculos con la sociedad que han venido deteriorándose.

Para hacer realidad esta visión de "compañero más capaz" por parte de la universidad, bien podría esta incorporar en sus estudiantes la competencia conectivista de Stephen Downes y George Siemens (ver por ejemplo Bongiovanni, 2012) ${ }^{5}$, que parte de las redes como las unidades para obtener conocimiento a través de asociaciones compartidas en una comunidad, al estar conectados al mundo en su forma más amplia, incluyendo la conexión que nos brinda el mundo cibernético de las tecnologías digitales, pudiese resultar una de las soluciones a nuestro problema de conocimiento.

Si aceptamos los postulados de Vygotsky de que la cultura y la comunicación interpersonal guían el desarrollo del niño, que las funciones mentales superiores se desarrollan a través de las interacciones sociales, derivando significados y afectando la construcción de "su conocimiento" a través de la internalización; y si a la vez aceptamos estar encontrando los elementos para intentar entender cómo el conocimiento se maneja dentro de nuestra cultura latinoamericana, o como podemos manejar el conocimiento conectivista a "la latinoamericana", estaremos iniciando el camino para establecer esquemas para la movilización eficiente de conocimiento entre nuestras universidades y su entorno social.

Serían esas interacciones sociales, y el tipo de información que fluye por esas redes en una comunidad, las que determinarán los tipos de conocimiento a los cuales serían reactivos los miembros de una determinada cultura y estructura social. Y si estos elementos los ponemos en el medio de toda la discusión que en forma recurrente nos recuerda que somos miembros de "la raza cósmica" (Vasconcelos, 1925), o que vivimos en "el laberinto de la soledad"

5 Adicionalmente remitimos al lector a trabajos originales de Downes (2011) y Siemens (2004). 
(Paz, 1950), o que saltamos "Del buen salvaje al buen revolucionario" (Rangel, 1982), por solo nombrar algunas aproximaciones al problema de nuestra unicidad, de las consecuencias de nuestro mestizaje, sin duda que deberíamos comenzar a visualizar algunas experiencias que podrían resultar sumamente interesantes para desentrañar nuestra relación cultural con la que ocurre a los habitantes de otras latitudes.

El conectivismo nos ofrece la posibilidad a los latinoamericanos de lograr en nosotros esa síntesis de la ciencia y la poesía que es la historia, como nos recuerda Octavio Paz, es poder hacer el trabajo del historiador que reconstruye con imaginación la sólida realidad de sus hallazgos, es poder salir exitosamente de lo mágico maravilloso, por poder manejar con facilidad la información exuberante que nos permite la tecnología. Relacionar la conectividad con la movilización de conocimiento tan necesaria en nuestra sociedad.

Pero para lograr esto, necesitamos un sistema educativo que apoye el proceso. Debemos encontrar la manera de insertar la conectividad y el conectivismo en nuestros docentes, y hacerlo aceleradamente, antes de que el hecho tecnológico vuelva a arroparnos y sigamos quedándonos en los bordes de la historia. Visto así, pareciera que pudiésemos encontrar en la técnica una manera de constituir un elemento connatural a nuestra cultura.

\section{La inserción de la ciencia y la tecnología en la universidad venezolana. Desde las aulas hasta el Parlamento}

En el siglo XVIII, el desarrollo de las ciencias experimentales cambió la orientación de los estudios: el hombre deja de ser el centro de preocupación y, en su lugar, se prestó mayor atención a la investigación sobre el mundo. La enseñanza verbal, como transmisión de un "corpus" doctrinal, fue sustituida por la observación, la experimentación y el "saber aprender" (Muscará, 2012). El nuevo 
modelo de generación de conocimiento con impacto social encuentra extraordinariamente positiva la estructura universitaria para su desarrollo, convirtiéndose así la universidad en el pináculo de los centros que generan conocimiento, como reporta ya en 1939 J. D. Bernal en La función social de la ciencia.

Sin embargo, la situación no se desarrolla de forma similar en nuestra parte del mundo. ${ }^{6}$ En la misma obra recién mencionada, Bernal escribe:

La ciencia en América Latina, hasta una fecha relativamente reciente, sufrió de la misma discapacidad que ba afligido la madre patria. En la época colonial, particularmente al principio, se bizo algo de bistoria natural y minería, pero pronto se desvaneció el interés y la revolución $y$ las guerras civiles que siguieron durante la mayor parte del siglo XIX no fueron propicias para el desarrollo de la ciencia. En el presente siglo, sin embargo, bajo la influencia de los Estados Unidos de América y del pensamiento liberal resurgente, un renacimiento en la ciencia de la cual puede esperarse mucho está empezando y ya, particularmente en México y Argentina, notables progresos se han realizado en medicina, biologia y arqueología.

Lo anterior nos muestra un cierto retraso en la universidad latinoamericana en lo relativo a la inserción de la investigación científica en su estructura y, por ende, en la capacidad de generar conocimiento.

Venezuela no escapa a esta situación. Aunque relativamente escasa, la actividad científica nunca ha dejado de cumplirse en el país, aunque en forma marginal (Roche, 1982). Desde la creación de la Universidad Real de Caracas por Cédula de Felipe V en 1721 hasta la universidad de los alrededores del inicio de la Segunda Guerra Mundial, el paradigma fundamental es la universidad transmisora de conocimiento. Alrededor de esta última época

6 La cultura española tiene algo que ver en todo esto, Roche ha ahondado en estas consideraciones (Roche, 1975). 
mencionada, con la llegada al país de hombres como Augusto Pi Suñer, escapado de la España republicana, o de venezolanos como Marcel Roche y Francisco de Venanzi, que recién retornaban de pasantías en Estados Unidos, se inicia un proceso que aspiraba convertir a nuestras universidades en generadoras de conocimiento.

Entre los años 1950 y 1975, surgen en rápida sucesión instancias que van logrando institucionalizar los estudios científicos en el país, tales como la Asociación Venezolana para el Avance de la Ciencia (AsoVAC), el Instituto Venezolano de Neurología e Investigaciones Científicas, (luego sin la N, IVIC), el Consejo Nacional de Investigaciones Científicas y Tecnológicas (CONICIT), para culminar en 1975, cuando se realiza el primer Congreso Venezolano de Ciencia y Tecnología. A lo largo de todo este proceso se desarrolla y consolida la labor de investigación en nuestras universidades, teniendo así una universidad transmisora y generadora de conocimiento.

La deseada institucionalización de la CyT en Venezuela va a encontrar en la universidad un extraordinario campo de cultivo. En particular entre los años 1958 y 1963, liderada por Francisco de Venanzi se va a producir una aceleración de este crecimiento institucional y presencial de la $\mathrm{Cy} T$ en las universidades públicas. Basta revisar las fechas de los discursos pronunciados por De Venanzi desde la Comisión Universitaria y luego como rector de la Universidad Central de Venezuela a lo largo de ese período (De Venanzi, 1961, 1963):

1. Instalación de la Facultad de Ciencias, 13 de marzo de 1958.

2. Palabras pronunciadas ante la Junta de Gobierno con motivo de presentar al Ejecutivo Nacional el Proyecto del Consejo Nacional de Investigación Científica, en representación de las Universidades Nacionales, la Asociación Venezolana para el Avance de la Ciencia y los gremios de profesionales, 9 de mayo de 1958. 
3. Apertura de la IX Convención Anual de la AsoVAC, 14 de junio de 1959.

4. Saludo a los participantes en el Seminario sobre la Organización de la Investigación Científica en Latinoamérica, acto inaugural, 3 de octubre de 1960.

5. Palabras pronunciadas con motivo de la instalación del Centro de Estudios del Desarrollo, 18 de marzo de 1961.

6. Inauguración de la Convención de Científicos, Educadores y Empresarios para propiciar el desarrollo económico del país, 5 de febrero de 1962.

Para conocer en mayor profundidad lo logrado en el desarrollo de la ciencia en estos años en el país se recomienda la lectura del libro de Roche, Descubriendo a Prometeo (1975). Algo similar ocurría para el sector educativo en general; un veloz crecimiento con dudas acerca de los resultados. ${ }^{7}$

Eran los tiempos de esperanza de los sesenta y setenta, esperanzas que circulaban tanto a nivel nacional como a nivel mundial. ${ }^{8} \mathrm{Un}$ hito importante fue el Primer Congreso Nacional de Ciencia y Tecnología realizado en 1975 (UCV, 1975).

Es nuestra opinión que el punto más alto de las esperanzas puestas en la CyT llega a inicios de la década de los ochenta. La AsoVAc, en alianza estratégica con la Asociación Interciencia, realiza en Caracas un Simposio llamado "La participación de la comunidad científica frente a las alternativas de desarrollo". En dicho evento,

\footnotetext{
Ver por ejemplo Hung de León y Piñango (1984), para la educación en general, o la cuantiosa producción de Orlando Albornoz (2013, por ejemplo).

8 A nivel mundial las Naciones Unidas adelantaron a través de la Unesco eventos como la Conferencia de las Naciones Unidas sobre Ciencia y Tecnología para el beneficio de las áreas menos desarrolladas se realizó en Ginebra en 1963 con impacto en toda América Latina (ver por ejemplo para el caso argentino Feld, 2010) y el resto del mundo.
} 
Luis Enrique Alcalá, entonces Secretario Ejecutivo del CONICIT, abogaba por una mayor coordinación por parte del Estado al comentar que en nuestro medio teníamos: "un Plan que no abarcaba todos los aspectos requeridos, cuyos actores y organizaciones 'no son muy coordinables' y que los entes encargados de orientar el sector contaban con un poder muy limitado", en contraste con las ponencias de científicos de Estados Unidos y Canadá (Piñango, 1982) donde se plantean los diversos mecanismos que se utilizaban en esos países para integrar a los científicos en la formulación de políticas.

Casi simultáneamente se estaba creando la Comisión de Ciencia y Tecnología de la Cámara de Diputados (CPCyTCD) del entonces Congreso de la República, también por iniciativa de los investigadores universitarios organizados en la AsoVAC, donde desde 1978 venían contactando a los dirigentes políticos e insistiendo en las ventajas de llevar el conocimiento científico a los lugares de toma de decisión de políticas públicas. Al poco tiempo, la Comisión inicia sus actividades y ya para el año 1983 comienza la edición de una serie de informes técnicos entre los que se incluyen títulos como: "Causas y consecuencias de la contaminación ambiental", "Problemática laboral de los egresados de las Facultades de Ciencias" y "Aspectos biosocioeconómicos en el desarrollo del venezolano", todos en el mismo año de 1983. Igualmente se difunde un "Boletín Informativo" que "persigue informar a los parlamentarios interesados en el tema sobre los estudios y las preocupaciones que mantienen ocupada a la Comisión" (CPCyTCD, 1983).

Este tratar de involucrar a los partidos políticos en la problemática de Cy'T lleva a la Carta de Sao Paulo (1990):

Los participantes en el Primer Seminario Latinoamericano de Dirigentes de Organismos Nacionales de Ciencia y Tecnología, realizado en Sao Paulo, Brasil, del 19 al 22 de febrero de 1990, ante la perspectiva y los desafíos que nos presenta la actualidad y que serán más importantes en el siglo XXI, en materia de ciencia y tecnología para un desarrollo integral de nuestras sociedades, hemos decidido emitir la presente.... 
En ese evento se presentaron ponencias venezolanas como "Partidos políticos, parlamento y la política científico tecnológica" (Ordóñez, 1990) donde se analizaba la participación de los partidos políticos y del poder legislativo en la definición de las políticas científicotecnológicas de los países.

Sin embargo, debemos reconocer que la mayoría de los esfuerzos por lograr este desarrollo de la capacidad de CyT provenían de la propia comunidad $\mathrm{Cy} \mathrm{T}$, y estaban orientados fundamentalmente a obtener mayor apoyo para el sector en la forma de incrementos presupuestarios. Así, el ya mencionado Boletín de la CPCyTCD plantea en un editorial de 1990:

Poca comprensión por parte del Estado de que los recursos asignados a Ciencia y Tecnología van a coadyuvar a la solución de muchos de los urgentes problemas nacionales en el campo de la agroindustria, energia y preservación del ambiente asi como también el mejoramiento general de las condiciones de vida del venezolano...", con el énfasis definitivamente puesto en "... la propuesta formulada por el Gobierno Nacional en el sentido de asignar el dos (2) por ciento del Producto Territorial Bruto a las actividades de CyT y en la Capacidad de la comunidad científica en cuanto a la óptima utilización y administración de los recursos que necesariamente, debe asignar el Estado en los próximos años al sector.

Es de hacer notar aquí que la gran mayoría de la "comunidad Cy'T" estaba constituida, y todavía sigue estándolo, por docentes universitarios.

Para 1992 creemos detectar el momento cuando se inician los reclamos hacia la ilusión de "solución a los problemas" creada por la CyT. La Comisión Presidencial para la Reforma del Estado (COPRE) trata el tema de la CyT, con una fuerte presencia, en su composición y temario, de miembros también provenientes del sector universitario (COPRE, 1992).

En la introducción al volumen de la COPRE sobre Ciencia y Tecnología en Venezuela, Machado-Allison (1992) escribe: 
Sin duda, "ciencia y tecnologia" están inscritas ya en el discurso, pero aún están ausentes en las politicas del Estado venezolano, en la actividad productiva, en los servicios públicos y, fundamentalmente, en la cultura global del país.

Lo que ocurrió posteriormente gira alrededor del Ministerio de Ciencia y Tecnología (MCT) que se orientó a la "consolidación de un sistema nacional de ciencia, tecnología e innovación que articule y sintonice los distintos actores sociales, dirigido a generar un crecimiento productivo con equidad con el lema "Ciencia y Tecnología para la Gente", "y que es solo el reflejo de hitos importantes del proceso político iniciado en 1998 tras la victoria electoral de Hugo Chávez: la Constitución de 1999, la promulgación de la Ley Orgánica de Ciencia, Tecnología e Innovación, en el 2001 y reformulada en 2005 (2005), así como el Plan Nacional de Ciencia, Tecnología e Innovación 2005-2030 (2005).

Sin embargo, en las difíciles relaciones entre el ejecutivo nacional y su proceso de "revolución bolivariana" y la universidad pública autónoma nacional, el apoyo real a la capacidad de CyT ha sido más bien disminuido y está recogido en el reciente libro de Albornoz (2013) que tiene por título La universidad: ¿Reforma o experimento? El discurso académico contemporáneo según la perspectiva de los organismos internacionales: los aprendizajes para la universidad venezolana y latinoamericana.

Aparte de desestimular al subsector de las universidades autónomas con restricciones presupuestarias, es poco lo que se ha alterado la institucionalidad de la Cy'T, o a sus instituciones fundamentales al interior de las universidades en este período. La capacidad de hacer ciencia sí se ha alterado, tanto en su equipamiento, como en sus posibilidades reales de colaboración interinstitucional. ${ }^{9}$

9 El tira y encoge político está muy bien recogido por los trabajos, tanto académicos como de opinión en los medios de dos investigadores venezolanos, Jaime Requena y Yajaira Freites. 
Una consideración es necesaria luego de este rápido examen del desarrollo de la universidad generadora de conocimientos del último medio siglo en Venezuela. A nivel internacional, ya desde 1978 cuando se producen los "Lineamientos de Pugwash para la cooperación científica para el desarrollo" (Pugwash executive committee, 1979) se observa un descreimiento en la capacidad de la $\mathrm{Cy}$ T para alterar los problemas sociales existentes. En el segundo párrafo del preámbulo puede leerse:

Cuando la Conferencia de las Naciones Unidas sobre Cienciay Tecnología para el beneficio de las áreas menos desarrolladas se realizó en Ginebra en 1963, se creía que una transferencia masiva de ciencia y tecnología desde los países desarrollados reduciría rápidamente la pobreza, el hambre y las enfermedades en los países en desarrollo en el mundo. Esta visión ha demostrado ser falsa.

Aunque esta situación trata de corregirse en la actualidad, sobre todo en Europa (ver por ejemplo el caso actual de la Unión Europea en Marco estratégico para la cooperación internacional en Ciencia y Tecnologia, 2008), la misma no ha cambiado en mucho hasta hoy, cuando observamos a la misma Fundación Dag Hammarskjöld publicando un libro donde se pregunta si se volvió irrelevante el sistema de las Naciones Unidas para el Desarrollo (Browne y Weiss, 2013).

Casi 25 años han pasado desde que se reúne la COPRE venezolana, pero la misma sensación pareciera estar presente en nuestra sociedad. Podemos repetir, como en el manifiesto de Pugwash, que la visión ha demostrado ser falsa, por lo menos desde los análisis de la Comisión para la Reforma del Estado hasta el presente. Pero cabe preguntarse si ha fallado la ciencia y la tecnología o si fue, por el contrario, la universidad, sobre todo aquella universidad pública que ha recibido sumas ingentes de dineros del Estado para desarrollar una capacidad Cy $\mathrm{T}$ sin que aún podamos sentirnos orgullosos y satisfechos con los resultados.

De ser la universidad quien falló, tendríamos entonces que preguntarnos cuáles son los factores que han impedido a la universidad 
cumplir con la promesa explícita de utilizar la Cy $\mathrm{T}$ para el desarrollo nacional.

El Estado clientelar, donde había dinero para todas las iniciativas (ver por ejemplo El caso Venezuela: Una ilusión de armonía, de Naim y Piñango,1984) es uno de los insumos que ha impedido que la universidad, como institución, participe en la movilización de conocimientos para la toma de decisiones de políticas públicas en el país, generando un estado de cosas que actúa como camisa de fuerza para que pueda la universidad impactar a la sociedad como quisiera, de manera que los esfuerzos realizados en el pasado tienden a diluirse y a perderse.

Este fenómeno se aprecia de forma más impactante a la hora de analizar los esfuerzos de nuestras universidades públicas por realizar Extensión. Los servicios de extensión tienden a estar aislados del resto de las estructuras universitarias y focalizados en las comunidades cercanas a la propia universidad. De manera que la tríada docencia, investigación y extensión marcha cada una por su lado, sin verdadera interacción (sin estar alineados dentro de estrategias más generales, dirían los expertos en administración de empresas) y aislados a su vez de las necesidades generales de la sociedad.

En este punto es conveniente una nota aclaratoria. En ningún caso nos referimos a los docentes-investigadores-extensores en sí mismos. Conocemos de excelentes docentes, excelentes docentesinvestigadores, excelentes docentes-extensores y hasta excelentes docentes-investigadores-extensores que hacen sus mejores esfuerzos para lograr excelentes resultados. Nos referimos aquí a la universidad como organización.

\section{Una tesis}

Creemos que la cultura universitaria, producto de su historia, y de su realidad, y la de la sociedad en que se encuentra situada, pudiese tener mucho que ver con esa falla que ahora se le reclama. También creemos que una revisión a fondo de esta situación pudiese llevar 
a un cambio de paradigma que generase los lenguajes, las estructuras y los nuevos mapas mentales que permitan a la universidad pública estar a la altura de las esperanzas que la sociedad ha depositado en ella.

Nuestra tesis es que la misión de la universidad pública es movilizar conocimiento para servir a la sociedad que la acoge y con ello contribuir a la mejora de las condiciones de vida de los integrantes de esa sociedad. Para ello la universidad debe organizarse de manera que su docencia, su investigación y su extensión sean mecanismos que ella utiliza para cumplir su función, y en esta tarea el docente universitario es solo parte del talento humano requerido, donde lo más importante son los subsistemas interrelacionados que la integran y que la conectan con la sociedad.

El verdadero desafío que se nos presenta es el de cómo puede la universidad pública movilizar conocimiento eficientemente para ayudar a resolver los problemas de la sociedad.

\section{El conocimiento y la toma de decisiones de Políticas Públicas. El caso universitario venezolano}

Al hablar de relación entre el Estado venezolano y la universidad, es bueno comenzar por lo que dice Orlando Albornoz en su obra La universidad, ¿reforma o experimento? (2013):

En V enezuela es el gobierno nacional el que abre y cierra instituciones, expande o restringe la matricula estudiantil, paga los sueldos y salarios de los miembros del personal docente y de investigación, fijándoles sus cotas laborales - excepto las del sector privado-; provee los fondos para que funcionen las instituciones del sistema de educación superior, avala los titulos y credenciales profesionales, designa las autoridades de prácticamente todas las instituciones del sector y define el ethos académico, estimulando o deprimiendo la atmósfera intelectual y académica, regulando los premios y castigos, según su leal saber y entender, como reza la antigua fórmula jurídica, al mismo tiempo que regula 
a los privados que quieren participar del mercado escolar, fijando el monto que pueden cobrar a los estudiantes. Esto es, Estado y gobierno se entremezclan en la dinámica del sistema nacional de la educación superior y ello ocurre desde la propia creación del sistema nacional, en 1827, cuando el Estado cré el monopolio del manejo de las universidades, fracturado solo en 1953, pero aún fuerte como para mantener el control derivado del papel omnipresente del estado y del gobierno de esta sociedad.

Sin embargo, la relación entre la universidad, en especial la universidad pública y el Estado venezolano es altamente deficiente e insatisfactoria para ambas partes a la hora de intercambiar conocimiento; y sobre todo, porque esa inadecuada relación es insatisfactoria para la misma, que asiste inerme al espectáculo de una institución que consume recursos para que la sociedad tenga conocimiento y saberes, y un Estado que no pareciera poder (o saber) utilizar todos esos saberes para brindar mejor calidad de vida a sus ciudadanos.

La percepción que de la universidad y en particular de sus postgrados y capacidades de investigación tiene el ciudadano latinoamericano promedio pudiese ser comparable a las visiones que se tienen sobre la conquista española de este continente, se mezclan entre una leyenda negra y una leyenda dorada. La visión negra de la universidad nos habla de que Latinoamérica no tiene buenas universidades ${ }^{10}$ y que las mismas deben renovarse. La visión dorada, en el mejor de los casos, nos recuerda permanentemente la lógica del jarrón chino: todos la admiran, pero nadie sabe qué hacer con ella. Y las investigaciones para vincular conocimiento y gestión académica apenas comienzan en nuestro medio (Rodríguez-Ponce et al., 2013).

Algunas preguntas clave que deberían responder investigaciones que aún aguardan por ser realizadas en nuestro país son, desde el

10 http://www.reportecatolicolaico.com/2013/07/\%C2\%BFse-puede-decir-que-lati noamerica-tiene-buenas-universidades/ recuperado el 14 de abril de 2014. 
lado de la demanda de conocimientos por el Estado, cuánto ha utilizado la Comisión de Ciencia y Tecnología en la Asamblea Nacional a la universidad para estudiar y decidir sobre problemas que haya considerado. De igual modo cabe preguntarse: cuál ha sido el conocimiento universitario movilizado por los diferentes ministerios de la rama ejecutiva de la administración vía grupos de trabajo, a la hora de decidir sobre políticas públicas orientadas a resolver los problemas que enfrenta el país. Como ejemplo para la reflexión pudiéramos recordar aquí las metas de los objetivos de desarrollo del Milenio ${ }^{11}$ propuestos por la ONU, y preguntar para cuántos de ellos se establecieron grupos de análisis conjuntos gobierno-universidad para enfrentarlos.

Esta situación de desconexión entre lo investigado por el mundo académico y las necesidades de la sociedad ha sido analizada por diferentes intelectuales en nuestro medio. Eduardo Devés Valdés, en el marco de la Conferencia de apertura del año académico en la Universidad de Puerto Rico, Recinto Río Piedras, Facultad de Estudios Generales, se refirió a "Las tareas de intelectuales y universitarios ante el estancamiento de América Latina hacia las redes y la internacional del conocimiento" (2006). Durante su disertación, organizada a lo largo de diez reflexiones, el ponente toca puntos tales como:

- Segunda reflexión: Sobre el estancamiento de América Latina y la necesaria equidad del poder mundial.

- Tercera reflexión: Sobre la baja producción de conocimiento en América Latina y su causa en los vicios de nuestra cultura académica.

- Cuarta reflexión: Sobre algunas potencialidades también existentes en nuestra cultura académica.

11 Los objetivos, sus metas específicas, así como una descripción general de la iniciativa se pueden leer en http://es.wikipedia.org/wiki/Objetivos_de_Desarrollo_del_Milenio 
- Quinta reflexión: Colaboración intelectual internacional y exportación de tecnología y cultura.

- Octava reflexión: Redes intelectuales y corredores de las ideas como formas de acción e interacción de la sociedad civil del conocimiento.

Como se puede deducir de los títulos mencionados, es mucho lo que tienen que realizar los académicos para la resolución de los problemas existentes. Sin embargo, el problema de si son los académicos, los políticos o las instancias llamadas a decidir sobre políticas públicas, tanto a nivel del ejecutivo como del legislativo, los que no utilizan adecuadamente el conocimiento existente en esas universidades, no se resuelve al fijar responsabilidades y sancionar a los responsables.

Aunque aceptamos que debe haber una relación bilateral entre universidad y Estado para movilizar conocimiento (con las necesarias estructuras intermediadoras) y que la misma no funciona adecuadamente en nuestro país, también es cierto que quien está llamada a tratar de entender la situación problemática y presentar posibles alternativas de solución (modelos que lleven a la acción "falsable"12) es la universidad, precisamente por su capacidad de ser "generadora de conocimiento para la toma de decisiones".

Desafortunadamente, en nuestro medio no es mucho lo que se encuentra en la literatura académica en el tema de "movilización de conocimientos entre la universidad y la sociedad", como podemos observar al analizar con cierto detalle las presentaciones realizadas durante el foro que tuvo por objetivo y título la ya mencionada "Movilización de conocimientos entre la universidad y la sociedad",

12 Falsabilidad: En filosofía de la ciencia, se entiende por falsabilidad a la propiedad que se verifica si se sigue, deductivamente, por modus tollendo tollens (del latín, modo que negando niega), que la proposición universal es falsa cuando se consigue demostrar mediante la experiencia que un enunciado observable es falso. Es un concepto central de la teoría epistemológica de Karl Popper conocida como falsacionismo. Recuperado el 14 de abril de 2014 de http:/ / es.wikipedia.org/wiki/Falsabilidad 
realizado con motivo de la LXIII Convención Anual de AsoVAC realizada en noviembre de 2013 por iniciativa de la Presidencia de esa institución y la Fundación InterConectados y con ponencias presentadas por docentes o personal administrativo de alto nivel de universidades tan prestigiosas como las universidades públicas Universidad Simón Bolívar (USB), Universidad de Carabobo (UC), Universidad de los Andes (ULA), Universidad Nacional Abierta (UNA), y privadas como la Universidad Católica Andrés Bello (UCAB), Universidad Tecnológica del Centro (UNITEC) y Universidad José Antonio Páez (UJAP).

En el foro se discutieron un total de once ponencias en las áreas de docentes universitarios, bibliotecas universitarias, investigación universitaria, extensión universitaria y la comunicación universitaria. Los objetivos específicos a desarrollar, planteados previamente a los ponentes para cada área, fueron:

- Los docentes universitarios: Cómo percibe la sociedad a la universidad. El docente como experto con conocimientos de utilidad pública. Cómo incorporarlo en el diseño e implantación de políticas públicas orientadas a la solución de problemas sociales. Cómo educar docentes para "la sociedad".

- Las bibliotecas universitarias: Cómo satisfacer las necesidades de información de la sociedad utilizando la capacidad instalada de las bibliotecas universitarias. El problema de la Alfabetización Informacional en la sociedad venezolana y el papel de las bibliotecas universitarias.

- La investigación universitaria. Cómo generar y gerenciar equipos de investigación para el estudio de problemas sociales complejos. La necesidad de integrar la investigación interdisciplinaria en el interior de la universidad.

- La extensión universitaria: Cómo poner las posibilidades de la universidad al servicio de toda la nación. Flujos de información para detectar problemas de la sociedad e instancias universitarias con posibilidades de apoyar la solución de esos problemas. 
- La comunicación universitaria: Cómo abrir canales bidireccionales para la comunicación entre universidad y sociedad (comunidades - centros de toma de decisiones de políticas públicas - intermediarias de conocimiento).

- Los intermediarios externos a la universidad: Cómo potenciar las relaciones entre consultores y profesionales expertos y la universidad. Cómo apoyar la intermediación.

Las láminas utilizadas por los diferentes ponentes se encuentran en la web (Foro AsoVAC, 2013) y permiten llegar a distintas conclusiones. Nuestra primera conclusión, expresada al final del evento, es que "la universidad mostrada en el foro distaba mucho de una donde esté toda la universidad tras la resolución de los problemas de conocimiento para el desarrollo del país", sobre la base de que la mayoría de las ponencias analizaba internamente la actividad universitaria correspondiente, describiendo sus fortalezas y debilidades y manteniendo casi sin mencionar a las otras actividades universitarias con las cuales podría lograr colaboraciones sinérgicas.

A pesar de la presencia de representantes de universidades públicas y privadas de toda la geografía nacional y de diferentes áreas del conocimiento y en diferentes posiciones al interior de la organización universitaria, cada sector invitado intentó presentarse y proyectarse a sí mismo, con muy pocas alusiones al trabajo en equipo o a los demás componentes de la estructura universitaria. Esta ausencia de visión holística es quizás la deficiencia más grande del actual paradigma universitario y que genera la competencia, muchas veces estéril, entre departamentos y unidades a la hora de resolver los problemas que les presenta la sociedad.

La situación detectada encuentra explicación en Pierre Bourdieu. Podemos visualizar a cada sector o espacio luchando por generar su propio campo y habitus, logrando así separarse progresivamente de los demás componentes de la organización universitaria, constituyéndose cada uno en reservorios aislados y protegidos, en lugar de conformar una sola organización eficiente. Un caso concreto: a mayor 
número de publicaciones mayor posibilidad de ascenso académico lo cual, unido a los estímulos de tipo económico como las "carreras de investigador", conducen a una búsqueda permanente de este tipo de meta por parte de los miembros de la academia. El capital cultural así generado va a ser utilizado posteriormente para diferenciarse de aquellos que no publican, no solo los individuos que están fuera del campo universitario, sino los que en el interior de él no participan de la carrera académica, como son los profesionales a cargo de labores administrativas, las cuales incluyen entre otras a las bibliotecas y las oficinas de comunicación social.

Lo anterior tiene varias consecuencias, la menor de la cuales no es que el investigador va a tender a investigar en aquello que ya conoce, y a la hora de colaborar con otros investigadores lo hará para reunir lo que ya ambos dominan y donde les resulta más fácil publicar. En el camino, y debido a los sistemas de incentivos y del mínimo esfuerzo, generados al haber convertido la publicación en un fin en sí misma debido al babitus académico, las investigaciones dejan de orientarse a resolver las necesidades de la sociedad y se tornan parte de los rituales preestablecidos por el mismo mundo académico.

Si aceptamos, por otra parte, que investigar los problemas sociales que actualmente enfrentamos representa comprender problemas complejos de difícil solución, podemos vislumbrar la dimensión del reto que se le presenta a los investigadores científicos y a la universidad como estructura organizativa a la hora de acometer la generación, movilización y aplicación de conocimientos en estas áreas problemáticas.

Solo eso podría explicar que a lo largo de once ponencias las estrategias orientadas a coordinar más eficientemente las estructuras universitarias existentes prácticamente brillaran por su ausencia, mientras el llamado a incrementar los presupuestos (en general) para que cada dependencia pudiese crecer a objeto de "cumplir más eficientemente su función" fueron casi el único común denominador de las presentaciones comentadas. 
Si a eso unimos el fenómeno de que la sociedad ha visto reducir sus expectativas para con la universidad hasta llegar al nivel de casi exclusivamente exigirle su función de "conferir títulos" (ver la primera ponencia en el foro), en lugar de asignar un papel más proactivo en la movilización de conocimientos para resolver sus problemas (esa función ha quedado casi exclusivamente al estamento político), podemos visualizar cómo el camino a recorrer para resolver la situación actual será largo y tortuoso.

Pero un evento no es suficiente para definir una tendencia. Debemos comentar aquí otros hallazgos basados en nuestra propia experiencia, y que vistos desde las máximas de la gestión del conocimiento, "el conocimiento requerido, en el lugar adecuado, en el momento justo" (De Brún, 2005) nos permitirán compensar por la ausencia de publicaciones en nuestro medio sobre este tema.

\section{Un caso particular: la biblioteca universitaria}

En el paradigma vigente, la biblioteca universitaria es una estructura al servicio de la universidad transmisora de conocimiento (tiene los libros y revistas que los profesores mandan a leer a los estudiantes) o de la universidad generadora de conocimientos (guarda las colecciones de revistas especializadas que consumen los investigadores en la universidad para adelantar sus tareas). Sin embargo, está poco preparada para atender demandas de conocimientos provenientes de posibles usuarios de la sociedad que rodea a las universidades (llámense estos consejos comunales, industrias, equipos deportivos o emprendedores), de donde resulta que con sus actuales recursos humanos y de infraestructura, la biblioteca no es una instancia proactiva en capacidad de proveer información "requerida, en donde se necesita y a tiempo" a los diversos usuarios extrauniversitarios que pudiesen necesitar conocimiento especializado. Esta misión se ha delegado al profesor universitario, que en forma simultánea con las otras demandas de docencia e investigación se supone debe hacer una labor de extensión, que a su 
vez está marcada por la "demanda implícita" de que sea "dentro de su área específica de conocimiento", todo lo cual se traduce en una deficiente atención a las demandas de conocimiento por parte de la sociedad.

Entender hacia dónde debe ir el cambio es difícil; por una parte, el paradigma de la biblioteca misma ha debido cambiar con la llegada de internet: ahora es más valiosa la conexión que pueda o no tener una biblioteca a las diferentes colecciones digitales existentes, en lugar de su propio "acervo documental". Por la otra, los énfasis ya no pueden estar al servicio de "la formación de colecciones" sino en apoyar a los usuarios en la búsqueda y procesamiento de esa información que se puede obtener "desde" la biblioteca, en lugar de "en" la biblioteca. Y esos usuarios no pueden estar ubicados exclusivamente "en el interior" de la universidad.

Esto nos obliga a considerar la necesidad de brindar servicios de información a la sociedad en la que está inmersa esa biblioteca, que requiere de información no necesariamente dependiente de la intermediación vía profesores. Este aspecto muy poco desarrollado en la actualidad, por no percibirse a sí misma la universidad como "movilizadora de conocimiento", tiene como base el hecho de que los recursos que invierte la sociedad para proveer de materiales a las bibliotecas universitarias no son en el fondo más que la búsqueda por tener a mano acumulaciones de conocimiento (información) que la misma sociedad debería estar en capacidad de utilizar cuando le fuese necesario. En el actual paradigma a ese conocimiento solo se puede acceder fundamentalmente a través de la intermediación de los docentes, y el docente está principalmente motivado a "generar conocimiento", base de su prestigio en su campo y gracias a su babitus, en lugar de distraer su atención y tiempo a resolver las necesidades de información de su entorno.

Por si no tuviera suficientes problemas, la biblioteca universitaria enfrenta uno adicional: 
Existe una gran paradoja: aunque los contenidos (información) se encuentran boy más que nunca disponibles (saber que existen) el hacerlos accesibles (poder apropiarse de ellos) amerita capacidades y destrezas precisamente para saber que existen y poder apropiarse de ellos. Esto se deriva de un hecho indudable: En el ambiente real del joven estudiante universitario, se puede contrastar la información acumulada en el ambiente familiar con la información obtenida del ambiente escolar. El problema en la época digital que apenas se inicia, es que la masa abrumadora de la información se encuentra fundamentalmente en la Web, y es alli donde los estudiantes deberán dirigir cada vez más su atención a fin de obtener la información requerida para generar el conocimiento necesario para funcionar en sociedad, tanto laboral como políticamente, considerando ambos roles desde el ámbito como ciudadano. (Ordóñez et al., 2013)

De lo anterior que el nuevo papel de las bibliotecas públicas universitarias consista en la intermediación del conocimiento en su seno y los individuos en la sociedad, no solo mediante la prestación de servicios de información oportunos y pertinentes, sino mediante el desarrollo de competencias para el uso eficiente de la información en sus usuarios; hablamos entonces de Alfabetización Informacional (ALFIN).

Los aspectos anteriores son factibles de lograrse mediante la interacción entre las bibliotecas universitarias y la docencia a ese nivel, tal como ha sido mencionado (Ordóñez et al., 2013), si se dispone de docentes que asumen su verdadero rol de mediadores del proceso y se apropian de la ALFIN como una actividad constante en su quehacer profesional. Esto implica que los docentes deben asumir un rol más activo en la promoción y mediación del uso intensivo de la información por parte de sus alumnos, para ello la ALFIN provee un conjunto de estrategias que apoyan la dinámica de su quehacer profesional. 
Una amplia discusión sobre este tema debe incluir, tanto la responsabilidad de las autoridades universitarias llamadas a liderar la tarea de insertar adecuadamente en sus estructuras administrativas las estrategias y competencias requeridas, como el esclarecimiento del papel de las bibliotecas universitarias, actualmente con demasiada frecuencia ancladas en la visión de ser específicamente responsables de la información almacenada en sus colecciones, cuando deberían tener un rol más activo en comunicar los nuevos avances y estrategias conectivas a sus poblaciones objetivo (Ordóñez, 2012).

\section{Otros casos de estudio}

A continuación mencionaremos otros casos que han sido analizados en una investigación en marcha (Adrián y Ordóñez, en preparación) donde analizamos tres situaciones vinculadas con el medio académico y la gestión de conocimiento a través de las interacciones de los estudiantes universitarios.

En el caso 1: el ámbito comunal, estudiantes universitarios realizan labores de servicio comunitario en sus zonas de residencia, interactuando con dirigentes y vecinos de esas comunidades, y donde se observaron las prácticas relacionadas con el uso de la información/ conocimiento para la resolución de problemas vinculados con el ámbito más natural y próximo al individuo, su propia comunidad.

En el caso 2: el ámbito académico propiamente dicho, en el que se observaron las prácticas relacionadas con el uso de la información/ conocimiento para la resolución de problemas asociados a lo largo de una materia de educación universitaria.

En el caso 3 (Adrián, 2010): el ámbito de la Participación Política, se observaron las prácticas relacionadas con el uso de la información/conocimiento para la resolución de problemas o situaciones asociadas al desempeño ciudadano o político de asociaciones estudiantiles (Federaciones de Centros Universitarios). 
Para esas tres situaciones se evaluó cómo se manejaba la situación de información/conocimiento de acuerdo a algunos estudios paradigmáticos presentes en la literatura ${ }^{13}$ (ver figura).

\section{Figura N. ${ }^{\circ} 3$}

Modelos de referencia para la identificación

de prácticas relacionadas con el conocimiento

(Adrián y Ordóñez, en preparación, a partir de Kwon

y Onwuegbuzie(2005), Oliver y Reddy (2006), Laise, Migliaresse

y Verteramo (2005), y Navarro, Colina y Sepúlveda (2007))

Factores determinantes de conductas

planificadas de uso de

información/conocimiento

(Kwon y Onwuegbuzie, 2005)

Aprendizaje

Interacción social

Diversión

Compras

Trabajo

Conexión con la comunidad

Pertinencia

Apresto tecnológico
Factores que promueven la cultura de conocimiento en las organizaciones

(Oliver y Reddy, 2006)

Liderazgo

Estructura

Evangelización

Comunidades de práctica

Sistema de recompensa

Distribución en el tiempo

Procesos

Reclutamiento

Infraestructura

Atributos físicos

\section{Cultura Organizacional de Información/Conocimiento}

Factores necesarios para la existencia

de relaciones organizacionales

intensivamente basadas

en el conocimiento

(Laise, Migliaresse y Verteramo, 2005)

Metas

Cultura

Reglas

Herramientas
Factores que condicionan los procesos de generación de conocimiento

(Navarro, Colina y Sepúlveda, 2007)

Trabajo en equipo

Captura intensiva de conocimiento externo

Relaciones de trabajo muy estrechos Elevada necesidad de producir

conocimiento nuevo

13 (Kwon y Onwuegbuzie (2005), Oliver y Reddy (2006), Laise, Migliaresse y Verteramo, S. (2005), y Navarro, Colina y Sepúlveda (2007). 
Entre las características que definen las prácticas organizacionales asociadas con la comunicación-información-conocimiento en los diferentes casos se pudo observar:

\section{EN EL CASO 1}

Ámbito comunitario: El grupo observado expresa desconfianza en el ente comunal en aspectos como: unidad representativa de los intereses de su comunidad, manejo precario de medios tradicionales de información-comunicación, manejo incipiente de medios electrónicos y ausencia casi total de uso de medios colaborativos. Expectativas poco retadoras en cuanto al uso y aprovechamiento de medios electrónicos dada por una tendencia Low Tech o escaso apresto tecnológico. Los vínculos entre los integrantes de los Consejos Comunales son débiles, y en lo que se refiere a la planificación y establecimiento de metas u objetivos de trabajo hay ausencia o debilidad en el establecimiento de una direccionalidad para las acciones, lo que se refleja en una ausencia de planificación. Aparte de la interacción social y el vínculo con la comunidad, no parece ser este un grupo con fortalezas en materia de información y conocimiento.

\section{EN EL CASO 2}

Ámbito Académico: Pudo observarse un uso intensivo y extensivo de medios electrónicos (Tendencia Hi Tech), expresado en el uso frecuente de medios colaborativos. Expectativas muy heterogéneas determinan el uso de los medios electrónicos de información, con predominancia del uso de la Red como medio de aprendizaje frente a las utilidades de diversión o interacción social, aunque estas también se mencionan con frecuencia en los relatos analizados. Los elementos menos mencionados en torno al uso de la Red, se vinculan al trabajo (asociado a la edad y condición de estudiantes de los relatores) y al vínculo con la comunidad, lo que parece indicar ausencia de motivación participativa en el uso de la Red. Es importante destacar, que la familiarización con el manejo que 
este grupo hace de las redes y sus recursos, provienen del entorno familiar o de amigos, no del entorno educativo que sin embargo norma y establece metas o utilidad esperada del uso de la información y el conocimiento. De los grupos estudiados es este el que presenta mayor presencia de factores de "cultura de conocimiento", es decir: motivadores como aprendizaje, interacción social, diversión, pertenencia y apresto tecnológico y elementos organizacionales (incipientes) como reclutamiento, propósito definido, reglas y herramientas.

\section{EN EL CASO 3}

Ámbito de la Participación Politica: se observó la ausencia casi absoluta de las federaciones de Centro en las Webs de sus respectivas universidades. Las Federaciones estudiantiles hacen uso limitado de la plataforma Web o de los Blogs para información general. El uso de las redes sociales tiene una finalidad casi exclusivamente noticiosa (avisos, convocatorias, etc.). La información que el grupo genera se encuentra dispersa y hay una aparente desvinculación entre organizaciones estudiantiles de distintas universidades. En este grupo si bien existen motivadores como la interacción social, la pertenencia y el apresto tecnológico se hace un uso limitado de los recursos disponibles, lo cual podría estar reflejando las debilidades organizacionales que de hecho aparecieron como inhibidores de las prácticas asociadas con información/conocimiento (ausencia de comunidades de práctica, evangelización, unidad de propósito, estrategias de reclutamiento, normas, entre otras).

De los casos analizados se desprende que uno de los aspectos que permitiría hablar de prácticas asociadas al uso de información/ conocimiento en el marco de una hipotética cultura de conocimiento es el comportamiento de sus miembros en lo que toca a reconocer y valorar la importancia de usar, conservar y compartir este bien o recurso. Hemos identificado brechas entre las prácticas observadas y el "deber ser" que plantea la literatura estudiada hasta el momento. 
En general, en los diferentes casos se observó poco arraigo de una cultura de trabajo en equipo; relaciones de trabajo debilitadas por factores como la desconfianza; o falta de claridad en objetivos comunes; alta valoración del recurso información y del hecho de estar informado que no se corresponde con el mínimo esfuerzo desplegado para generar, sistematizar y compartir información; metas y reglas poco claras (y no necesariamente compartidas); niveles heterogéneos de acceso y manejo de la tecnología.

Es necesario hacer la salvedad de que las organizaciones observadas son de carácter incipiente: comunidades en pleno proceso de organización, grupos universitarios agrupados en torno a un curso académico y organizaciones estudiantiles con elevados niveles de rotación. La utilidad de estos casos radica en poder evidenciar cómo se presentan (o no) las características descritas como ideales o al menos deseables en la exitosa gestión del conocimiento, en los modelos normalizados utilizados para el contraste.

El hecho de que en todos los casos la intervención o acción observada estuviera mediada por los estudiantes universitarios nos habla del poco impacto que la experiencia académica tiene en generar las competencias requeridas por la nueva sociedad del conocimiento y la urgencia de que estos aspectos sean revisados en los currículos universitarios.

Una consecuencia negativa es que de estos jóvenes estudiantes universitarios saldrán los dirigentes políticos de un país que no dispondrá de líderes en capacidad de movilizar eficientemente conocimiento a la hora de tomar decisiones de políticas públicas, por no haber recibido en la universidad las herramientas y competencias necesarias para ello.

No es fácil movilizar conocimientos en una sociedad para incidir en las políticas públicas. Como cualquier observador puede atestiguar, los prejuicios y desconfianzas son recíprocos entre las dos 
comunidades principalmente involucradas, a saber: los científicos y los políticos. Max Weber tocó el tema en El político y el científico (1919) y recientemente en dos artículos el periódico The Guardian del Reino Unido se enfocan las dificultades de esta relación de una manera original. Milman (2013) publicó una nota con el título Las principales 20 cosas que los politicos deben saber sobre la elaboración de políticas públicas y poco después Tyler (2013) respondió con Las principales 20 cosas que los científicos deben saber acerca de la elaboración de políticas públicas. Los 40 elementos se presentan en la tabla a continuación.

\section{“Alteridades” entre políticos y científicos}

\begin{tabular}{|c|c|}
\hline $\begin{array}{l}\text { Lo que los políticos deben saber } \\
\text { sobre la ciencia }\end{array}$ & $\begin{array}{l}\text { Lo que los científicos deben saber } \\
\text { sobre las políticas públicas }\end{array}$ \\
\hline $\begin{array}{l}\text { 1. Las diferencias y el azar producen } \\
\text { variaciones } \\
\text { 2. Ninguna medida es exacta } \\
\text { 3. El sesgo es generalizado } \\
\text { 4. El tamaño de la muestra, mientras } \\
\text { más grande mejor } \\
\text { 5. Una correlación no implica causalidad } \\
\text { 6. Una regresión a la media puede inducir } \\
\text { a error } \\
\text { 7. Extrapolar más allá de los datos es } \\
\text { arriesgado } \\
\text { 8. Cuidado con la falacia de la tasa basal } \\
\text { 9. Los controles son importantes } \\
\text { 10. El muestreo al azar evita sesgos } \\
\text { 11. Busque estudios replicados, no pseu- } \\
\text { doreplicación. } \\
\text { 12. Los científicos son humanos } \\
\text { 13. La significancia es significativa } \\
\text { 14. Separe el no-efecto, de la no signifi- } \\
\text { cancia } \\
\text { 15. El tamaño del efecto tiene importancia }\end{array}$ & $\begin{array}{l}\text { 1. Definir políticas es realmente difícil } \\
\text { 2. Ninguna política es perfecta } \\
\text { 3. Los que hacen las políticas también } \\
\text { pueden ser expertos } \\
\text { 4. Los que hacen las políticas no son un } \\
\text { grupo homogéneo } \\
\text { 5. Los políticos también son humanos } \\
\text { 6. Las decisiones de política están sujetas } \\
\text { a extensos análisis } \\
\text { 7. Iniciar políticas desde cero casi nunca } \\
\text { es la opción } \\
\text { 8. En una política hay algo más que evi- } \\
\text { dencia científica } \\
\text { 9. La economía y el derecho son los que } \\
\text { llevan la batuta en el asesoramiento } \\
\text { normativo } \\
\text { 10. La opinión pública cuenta } \\
\text { 11. Los hacedores de políticas entienden } \\
\text { de incertidumbre } \\
\text { 12. El Parlamento y el Gobierno funcio- } \\
\text { nan de maneras diferentes. }\end{array}$ \\
\hline
\end{tabular}


16. Los datos pueden "hablar por sí solos" o ser seleccionados para demostrar lo que se busca

17. Las medidas extremas pueden confundir

18. La relevancia de los estudios limita su generalización

19. Los sentimientos limitan la percepción de riesgo

20.Las interdependencias de las variables alteran los riesgos
13. Definir políticas públicas y hacer política no son la misma cosa

14.Los Parlamentos pueden tener excelentes sistemas de asesoría científica

15.Las políticas públicas y la ciencia operan en diferentes escalas de tiempo

16. No existe "un ciclo de las políticas públicas"

17.El arte de definir políticas públicas es una ciencia en desarrollo

18. Se debe distinguir entre "política para la ciencia" de "ciencia para las políticas"

19.Los que hacen políticas no están interesados en la ciencia per se.

20. "Necesitamos más investigación" es la respuesta incorrecta

Las diferentes culturas del mundo académico y político son en parte responsables de las dificultades a la hora de movilizar conocimientos. También lo son las "culturas nacionales". En lo que respecta a la cultura del país, sería posible vincular el tema de las prácticas asociadas al uso de información/conocimiento en las organizaciones estudiadas con elementos de la cultura de nuestra sociedad.

¿Incide la cultura nacional en el éxito o fracaso de las iniciativas de gestión de conocimiento en sus organizaciones? La respuesta está en la literatura. Hofstede (1997) incluye a Venezuela en el marco de un estudio destinado a correlacionar cuatro de las cinco dimensiones culturales descritas por él, a saber:

1. Distancia hacia el poder, representada en el comportamiento hacia figuras de poder;

2. Índice de individualismo, representado por la tendencia hacia el colectivismo o al individualismo; 
3. Índice de "masculinidad" que expresa el grado en que los roles por género están claramente diferenciados, con valores predominantes como el éxito material y el progreso, seguridad, agresividad y ambición o simpatía por la fortaleza, entre otros; e

4. Índice de rechazo a la incertidumbre, definido como el grado en que los miembros de una cultura se sienten amenazados por situaciones desconocidas o de alto nivel de incertidumbre.

En el estudio, Venezuela fue caracterizada como un país con:

1. Alta valoración del poder (prevalece el poder sobre el derecho; riqueza, poder y estatus son elementos considerados importantes, entre otras características).

2. Bajo índice de individualismo, lo que por oposición significa una alta valoración del colectivismo (se evita la confrontación, alta valoración del sentido de pertenencia, rol dominante del estado en la economía, administración entendida como cosa de grupos, entre otras características).

3. Alto índice de masculinidad (alta valoración del éxito material y el progreso, seguridad, agresividad y ambición o simpatía por la fortaleza, entre otras características).

4. Alto índice de rechazo a la incertidumbre y al riesgo (necesidad de reglas, temor ante situaciones ambiguas o desconocidas, incertidumbre percibida como amenaza constante que debe ser combatida, entre otras características).

Evidentemente, algunas de estas características "nacionales" inciden en la forma como se gestiona el conocimiento en las organizaciones y se encuentran presentes en los casos analizados, como lo ha encontrado Granell para las empresas venezolanas (2000). En un anterior trabajo (Ordóñez, 2012) se considera ampliamente el tema cultural en el marco del uso de la información para la participación y la superación de la pobreza. Si bien las culturas nacionales influyen 
en el comportamiento colectivo, se debe profundizar en el estudio del entorno que las determina, ya que las organizaciones mantienen características propias de su sector o rama de actividad (tecnologías, políticas, normas, etc.) que permiten establecer prácticas específicas, maneras de hacer las cosas y una cultura particular en cada una de ellas.

No es de extrañar entonces que ni la universidad a su interior, ni la sociedad, entendida esta como acumulación de organizaciones, estén en situación adecuada para la movilización de conocimientos eficientemente entre ellas. Pero si entendemos lo que ocurre y sus razones, al menos parcialmente, deberíamos ser capaces de utilizar el modelo así generado como estímulo para generación de respuestas que nos permitan superar la situación problemática. Creemos que esas respuestas deben venir desde la educación y la conectividad que permiten las tecnologías de información y comunicación.

A partir de los ya mencionados postulados de Vygotsky y del paradigma conectivista de Stephen Downes y George Siemens, pareciera que podemos estar encontrando los elementos para intentar entender cómo el conocimiento se maneja dentro de nuestra cultura latinoamericana, o cómo podemos manejar el conocimiento conectivista a "la latinoamericana".

Porque, serían esas interacciones sociales, y el tipo de información que fluye por esas redes en una comunidad, las que determinarán los tipos de conocimiento, "el conocimiento" al cual serían reactivos los miembros de una determinada cultura, de una determinada estructura social. Una de esas experiencias que creemos posible en nuestro sociedad, o mejor dicho, en los individuos que la componen, es la de asumir la conectividad, el estar conectados al mundo en su forma más amplia, incluyendo la conexión que nos brinda el mundo cibernético de las tecnologías digitales, de una forma mucho más natural por nuestra condición mestiza que la que ocurre a los habitantes de otras latitudes. 
Mientras eso ocurre seguiremos contribuyendo con el mantenimiento del círculo vicioso según el cual individuos inadecuadamente preparados para la colaboración, tanto por razones culturales como educativas, generan instituciones poco preparadas para esa colaboración, tanto por el inadecuado procesamiento de la información a nivel individual, como por la ineficiente movilización de conocimientos entre y al interior de las organizaciones.

\section{Recomendaciones para la toma de decisiones}

Los Rose escriben en su obra Science and Society (1969) que si bien es cierto que mucho de lo que es hoy en día el mundo moderno ha sido creado por la ciencia, también es cierto que mucho de lo que es hoy en día la ciencia ha sido moldeado por los requerimientos y constricciones que le han sido impuestos por la sociedad en las cuales se desarrolla. En el recorrido que hemos realizado desde los orígenes de la universidad hasta la necesidad de que la universidad pública latinoamericana actúe como una verdadera movilizadora de conocimientos en nuestras sociedades, debemos aceptar que debe desarrollar, tanto al interior como fuera de sí misma, las exigencias y orientaciones que lo logren.

El primer paso es asegurar que los egresados universitarios resulten consumidores naturales del conocimiento universitario, bien sea como profesionales en ejercicio, o como emprendedores a todos los niveles, o como políticos en las legislaturas y demás espacios de tomas de decisiones de políticas públicas. Y un buen lugar para comenzar la tarea es el nivel de formación de los doctores, con su potencial de iniciar las transformaciones requeridas al interior de la propia universidad. Es necesario definir desde un principio los parámetros que medirán ese impacto y comenzar el proceso de control y seguimiento permanente de los mismos.

Esta experiencia ya ha comenzado y hablaré en detalle sobre ella en un próximo artículo. 


\section{Bibliografía}

Adrián, S. (2010). Uso de la información, el conocimiento y el aprendizaje en la formulación de estrategias para promover la participación politica de los jovvenes. [Doctorado en Ciencias Sociales y Humanidades. Seminario de Participación]. Recuperado de http://es.wi kieducator.org/images/8/86/Papel_Estrategico_Informaci $\%$ C $3 \%$ B3n_Sixta.pdf

Albornoz, O. (2013). La universidad ¿Reforma o experimento? El discurso académico contemporáneo según la perspectiva de los organismos internacionales: los aprendizajes para la universidad venezolana y latinoamericana. Caracas: IESALC-Unesco.

Bernal, J. D. (1939). The Social Function of Science. Massachusetts: MIT Press Paperback.

Bongiovanni, P. (2012). 10 ideas poderosas acerca del aprendizaje conectivo. Recuperado de http://www.educacontic.es/blog/ 10-ideas-poderosas-acerca-del-aprendizaje-conectivo

Bourdieu, P. (1998). La distinción: criterio y bases sociales del gusto. Madrid: Taurus.

Bourdieu, P. \& Passeron, J.-C. (2009). Los herederos: Los estudiantes y la cultura. Buenos Aires: Siglo XXI.

Browne, S. \& Weiss, T. G. (2013). Is the UN development system becoming irrelevant? In Development Dialogue Paper, N. ${ }^{\circ}$. Recuperado de http://www.dhf.uu.se/wordpress/wpcontent/uploads/2013/12/dd-paper_no4.pdf

Brún, De, C. (2005). ABC of Knowledge Management. Recuperado de http://www.fao.org/fileadmin/user_upload/knowledge/ docs/ABC_of_KM.pdf 
Carta de Sao Paulo (1990). Primer Seminario Latinoamericano de Dirigente de Organismos Nacionales de Ciencia y Tecnología. Sao Paulo, Brasil. Recuperado de http://issuu.com/lordonezv /docs/carta_de_sao_paulo_y_pp_y_pcyt

Comisión Permanente de Ciencia y Tecnología. (1983). Boletín informativo, 1(1), marzo-mayo (s.p.).

Comisión Permanente de Ciencia y Tecnología. (1983). Boletín informativo, 8(1), enero-abril (s.p.).

Comisión Presidencial para la Reforma del Estado. (1992). Ciencia y Tecnología en Venezuela: un reto, una esperanza. (Vol. 12). Caracas: Comisión Presidencial para la Reforma del Estado.

Devés Valdés, E. (2006). Las tareas de intelectuales y universitarios ante el estancamiento de América Latina hacia las redes y la internacional del conocimiento. Recuperado de http://umbral.uprrp.edu/ ?q=Las_tareas_de_intelectuales_y_universitarios_ante_el_ estancamiento_de_America_Latina

Downes, S. (2011). The Massive Open Online Course in Theory and in Practice. Recuperado de http://www.slideshare.net/Downes/ xmooc-the-massive-open-online-course-in-theory-and-inpractice

Feld, A. (2010). La ciencia y la tecnología como objetos de reflexión en América Latina: ideas, actores, instituciones (1960-1970) (presentado en ESOCITE 2010). Recuperado de http://www.esocite2010. escyt.org/download_ponencia.php?file=Feld1278082626.doc $\&$ Code $=$ vf47aafdg7tvi9d5dujlpbiyg6q1jhl9y5v70n4i12zbh y2w0d8zy70psmkc

Granell, E. (2000). ¿Cómo crear una cultura para gerenciar el conocimiento? Gerencia del conocimiento. Potenciando el capital intelectual para crear valor. Caracas: Fondo Editorial del Centro Internacional de Educación y Desarrollo. 
Hofstede, G. (1997). Cultures and organizations: software of the mind. New York: McGrawHill [también puede visitar su página web Geert Hofstede Analysis en http://www.cybor link.com/besite/hofstede.htm]

Hung de León, L. \& Piñango, R. (1984). ¿Crisis de la educación o ciris del optimismo y el igualitarismo? Caracas: Ediciones IESA.

Inglehart, R. \& Welzel, Ch. (2010). Changing Mass Priorities: The Link Between Modernization and Democracy. Perspectives on Politics, 8, 551-567.

Katz, R. \& Mair (1995). Changing models of party organization and party democracy, Party Politics, 1, 1-5. Recuperado de http://politica comparata.files.wordpress.com/2011/03/katz-and-mair-1995changing-models-of-party-organization.pdf

Kwon, N. \& Onwuegbuzie, A. (2005). Modeling the factors affecting individuals' use of community networks: A theoretical explanation of community-based information and communication technology use. Journal of the American Society for Information Science and Technology, 56(14), 1525-1543.

Laise, D.; Migliaresse, P. \& Verteramo, S. (2005). Knowledge organization design: a diagnostic tool. Human systems management, 24, 121-131.

Leistner, F. (2010). Mastering organizational knowledge flow: how to make knowledge sharing work. New Jersey: Wiley and SAS.

Machado-Allison, C. E. (1992). Ciencia y tecnología en el contexto de la reforma del Estado, ciencia y tecnología en Venezuela: un reto, una esperanza (Vol. 12). Caracas: Comisión Presidencial para la Reforma del Estado.

Martínez García, J. S. (1998). Las clases sociales y el capital en Pierre Bourdieu: Un intento de aclaración (Comunicación presentada en el VI Congreso de Sociología de la FES). Recuperado de http://josamaga.webs.ull.es/Papers/clase-bd-usal.pdf 
Meulen, B., Van Der, \& Arie, R. (1998). Mediation in the Dutch Science System. Research Policy, 27, 757-769.

Meulen, B., Van. Der. (2003). New roles and strategies of a research council: intermediation of the principal-agent relationship. Science and Public Policy, 30, 323-336.

Milman, O. (2013). Top 20 things politicians need to know about science. The Guardian. Recuperado de http:/www.theguardian. com/science/2013/nov/20/top-20-things-politicians-needto-know-about-science

Muscará, F. (2012). Paradigmas universitarios a través del tiempo. Recuperado de http://www.um.edu.ar/math/maestria/ paradigmas.htm

Naim, M. \& Piñango, R. (1984). El caso Venezuela: una ilusión de armonía. Caracas: Ediciones IESA.

Navarro, K., Colina, B. \& Sepúlveda, J. (2007). Microcomunidades académicas de conocimiento: el núcleo agropecuario de la Universidad del Zulia. Revista Venezolana de Gerencia, 12(9), 307-350.

Oliver, S. \& Reddy, K. (2006). How to develop Knowledge culture in organization? A multiple case study of large distributed organizations. Journal of Knowledge Management, 10(4), 6-24.

Ordóñez, Vela, L. (1990). Partidos politicos, parlamento y la politica cientifica y tecnológica. (Ponencia presentada ante el Primer Seminario Latinoamericano de Dirigentes de Organismos Nacionales de Ciencia y Tecnología). Recuperado de http://issuu.com/lordonezv/docs/carta_de_sao_paulo_y_ pp_y_pcyt

Ordóñez Vela, L. A. (2012). Cultura participativa y conectivismo: algunos retos para la investigación latinoamericana. En Diálogos y desafios euro-latinoamericanos: Ensayos sobre cooperación, derecho, 
educación y comunicación. Recuperado de http://issuu.com/ lordonezv/docs/retos_para_la_investigaci_n_sobre_conec tivismo\#

Ordóñez, L., Adrián, S. \& Goncalves, N. (2013). Información o comunicación: El falso dilema de las bibliotecas universitarias, ARJÉ Revista de Postgrado FACE-UC, 7(12), 337-360.

Paz, O. (1992). El laberinto de la soledad. México: Fondo de Cultura Económica.

Piñango, R. (1982). Relatoría de la sesión orientación orgánica de la ciencia. En M. Aguilera, V. Rodríguez Lemoine y L. Yero (Ed.). La participación de la comunidad cientifica frente a las alternativas de desarrollo (pp. 123-126). Caracas: Asociación Venezolana para el Avance de la Ciencia.

Pugwash Executive Committee. (1979). Pugwash Guidelines for International Scientific Cooperation for Development, Development Dialogue, 1, 71-80. Recuperado de http:/ /www.dhf. uu.se/pdffiler/79_1.pdf

Rangel, C. (1982). Del buen salvaje al buen revolucionario. Caracas: Monte Ávila Editores.

Roche, M. (1975). Descubriendo a Prometeo. Caracas: Monte Ávila Editores.

Roche, M. (1982). Apuntes para una historia de la ciencia en Venezuela (desde su inicio hasta 1950). En M. Aguilera, V. Rodríguez Lemoine y L. Yero (Eds.). La participación de la comunidad cientifica frente a las alternativas de desarrollo (pp. 13-42). Caracas: Asociación Venezolana para el Avance de la Ciencia.

Roche, M. (1987). Los estados desunidos de América. En M. Roche. Mi compromiso con la ciencia (pp. 109-111). Caracas: Monte Ávila Editores. 
Rodríguez-Ponce, E.; Pedraja-Rejas, L.; Araneda-Guirriman, C.; Rodríguez-Ponce, J. (2013). La relación entre la gestión del conocimiento y la gestión académica: un estudio exploratorio en universidades chilenas. Interciencia, 38, 2, 88-94. Recuperado de http://www.redalyc.org/pdf/339/339269 50006.pdf

Romo Pedraza, A. (s. f.). El enfoque sociocultural del aprendizaje de Vygotsky. Recuperado de http://www.monografias.com/ trabajos10/enso/enso.shtml\#ixzz2q6S1EjYo

Rose, H. \& Rose, S. (1969). Science and Society. Londres: Penguin Books.

Shaxson, L. \& Bielak, A. T. et al. (2012). Expanding our understanding of $\mathrm{K}+(\mathrm{KT}, \mathrm{KE}, \mathrm{KTT}, \mathrm{KMb}, \mathrm{KB}, \mathrm{KM}$, etc.) A concept paper emerging from the $\mathrm{K}+$ conference held in Hamilton. Ontario: Naciones Unidas.

Siemens, G. (2004). Connectivism: A Learning Theory for the Digital Age. Recuperado de http://citeseerx.ist.psu.edu/viewdoc/ download?doi $=10 \cdot 1 \cdot 1 \cdot 87.3793 \& \mathrm{rep}=$ rep1\&type $=$ pdf

Taylor, M. C. (2010). Crisis on Campus: A bold plan for reforming our colleges and universities. New York: Knopf Publishing Group.

Tyler, Ch. (2013). Top 20 things scientists need to know about policy-making. The Guardian. Recuperado de http://www. theguardian.com/science/2013/dec/02/scientists-policygovernments-science?CMP $=$ twt_gu

Universidad Central de Venezuela. (1975). La universidad y el desarrollo cientifico y tecnológico: Papel de trabajo elaborado con motivo del Primer Congreso Nacional de Ciencia y Tecnología. Caracas: Ediciones del Rectorado. 
Unión Europea. (2008). Marco estratégico para la cooperación internacional en Ciencia y Tecnología. Recuperado de http://europa.eu/ legislation_summaries/research_innovation/general_frame work/ri0006_es.htm

Vasconcelos, J. (1925). La raza cósmica: Misión de la raza iberoamericana. [Para una rara versión hablada véase https://www.youtube. com/watch?v=Kdeepo_446k]

Venanzi, F., de. (1961). Palabras para una gestión universitaria: Discursos 1958-1961. Caracas: Universidad Central de Venezuela.

Venanzi, F., de. (1963). Palabras para una gestión universitaria: Discursos 1961-1963. Caracas: Universidad Central de Venezuela.

Venezuela. [Leyes, etc.]. (2005). Ley orgánica de ciencia, tecnología e innovación. Recuperado de http://www.uc.edu.ve/uc_ empresas/LOTIC.pdf

Venezuela. (2005). Plan Nacional de Ciencia, Tecnología e Innovación: Construyendo un futuro sustentable. Recuperado de http://es. slideshare.net/estraluna08/plan-nacional-de-ciencia-tecno loga-e-innovacin-20052030

Weber, M. (1972). Elpolitico y el cientifico. Madrid: Alianza Editorial. 


\section{Dr. Luis Arnaldo Ordóñez Vela}

Nacido en Maracay, Venezuela. Tiene una licenciatura en Química por la Universidad Central de Venezuela; doctor en Bioquímica egresado del Instituto Tecnológico de Massachusetts, Estados Unidos; culminó el Programa Avanzado de Gerencia (PAG) en el Instituto de Estudios Superiores de Administración (IESA), de Caracas, Venezuela.

Es el presidente de InterConectados y se desempeña como docente fortuito de varias universidades en cursos de pregrado y postgrado, entre los que se han contado el doctorado de Ciencias Sociales y Humanidades de la USB (Caracas, Venezuela), Seminario sobre la Participación; del doctorado de Educación de la Universidad Central de Venezuela; del Seminario de Investigación; y del doctorado de FACES, UC (Valencia, Venezuela, Organizaciones con Pertinencia Social). Igualmente dicta Cursos Abiertos Masivos En Línea (CAMEL) entre los que destacan "Participación Ciudadana", "Estructuración de Problemas Complejos" e "Investigaciones Colaborativas en Línea".

Sus intereses actuales de investigación giran alrededor de los determinantes de la participación en la sociedad y el impacto que tienen en la misma la presencia acelerada de las tecnologías digitales, así como el rol que juega la información en los mecanismos decisionales en nuestro medio latinoamericano y la movilización de conocimientos entre la universidad y la sociedad latinoamericana. 
La universidad y la movilización de conocimientos en la sociedad:

El caso venezolano

Sus publicaciones recientes son:

- Aprendiendo a aprender en la web: Una introducción al aprendiraje conectivista.

- Información o comunicación: el falso dilema de las bibliotecas universitarias.

- La promesa de internet y la participación ciudadana.

- Cultura participativa y conectivismo en la educación superior.

- Los partidos politicos venezolanos y su estrategia de aproximación a internet: una mirada no tecnológica.

- Variables psicosociales y microcréditos como estrategias de luchas contra la pobreza, entre muchas otras.

Correo electrónico: lordonezv@gmail.com

Recibido: 10/01/2015

Aprobado: 07/04/2015

Ciencia y Sociedad 2015; 40(2): 233-283 283 
